\title{
Reunification of a split sciatic nerve
}

\author{
Brendan Huang ${ }^{1}$, Tess Decater, Joe Iwanaga ${ }^{1}$, Aaron S. Dumont ${ }^{1}$, R. Shane Tubbs ${ }^{1,2,3,4}$ \\ ${ }^{1}$ Department of Neurosurgery, Tulane Center for Clinical Neurosciences, Tulane University School of Medicine, New Orleans, LA, ${ }^{2}$ Department of \\ Structural \& Cellular Biology, Tulane University School of Medicine, New Orleans, LA, ${ }^{3}$ Department of Neurosurgery and Ochsner Neuroscience \\ Institute, Ochsner Health System, New Orleans, LA, USA, ${ }^{4}$ Department of Anatomical Sciences, St. George's University, St. George's, Grenada, West \\ Indies
}

\begin{abstract}
Sciatic nerve (SN) variaitons can result in interesting clinical presentations. We identified a SN variant that does not fit into preexisting classification schemes. In an adult male cadaver, the SN was found to divide proximally and partly exit through the piriformis muscle. Distal to the piriformis, the two parts of the SN were reunited. Although apparently extremely rare, such a finding should be added to the archives of anatomical variations.
\end{abstract}

Key words: Anatomy, Anatomic variation, Cadaver, Sciatic nerve

Received April 20, 2020; Revised May 6, 2020; Accepted May 13, 2020

\section{Introduction}

The sciatic nerve (SN) is the largest nerve in the human body (approximately 2 centimeters in diameter) [1]. It arises from the ventral rami of L4-S3, is comprised of both motor and sensory components, and supplies the majority of the lower limb via its tibial nerve (TN) and common fibular nerve (CFN) components.

In their study of 120 cadavers, Beaton and Anson [2] were the first to characterize the different anatomical variants of SN bifurcation in relation to the piriformis muscle (PM). They concluded that the vast majority of their cadavers demonstrated, bilaterally, an unbranched SN that exits distally below the piriformis (type A) - this is considered the normal relationship between the nerve and muscle [3]. The most prevalent $\mathrm{SN}$ variant demonstrates branching proximal to the piriformis and exits through the muscle as the CFN while the TN arises inferiorly (type B) [2]. The second most common variant (type C) demonstrates the CFN and TN

\footnotetext{
Corresponding author:

Joe Iwanaga (i)

Department of Neurosurgery, Tulane Center for Clinical Neurosciences, Tulane University School of Medicine, New Orleans, LA 70112, USA

E-mail: iwanagajoeca@gmail.com
}

arising superiorly and inferiorly to the undivided PM, respectively [4]. Types D-F showcase other variations that are seen less frequently $[2,5]$. Herein, we report a unique variant of the SN.

\section{Case Report}

A variant SN was found during the routine dissection of a male Caucasian cadaver aged 72-years-old at death. The right $\mathrm{SN}$ was split by the PM into two roots, a superior CFN part and an inferior TN part (Fig. 1). The superior root was found to pierce the piriformis and the inferior root traveled inferior to the inferior border of the piriformis (infrapiriformis foramen). Distal (approximately $3 \mathrm{~cm}$ ) to the piriformis, the CFN and TN were united by a communicating branch (Cb) descending superomedial to inferolateral in an oblique manner. The $\mathrm{Cb}$ was approximately $1 \mathrm{~cm}$ in width by $3 \mathrm{~cm}$ in length. Distal to this $\mathrm{Cb}$, the CFN and TN continued toward the popliteal fossa and were distributed in normal fashion to the anterior/lateral and posterior compartments of the leg, respectively. No atrophy or other pathology was noted in any muscles of the leg and no other anatomical variations of the left or right lower limbs (including gluteal region) were noted.

\section{Copyright (c) 2020. Anatomy \& Cell Biology}

This is an Open Access article distributed under the terms of the Creative Commons Attribution Non-Commercial License (http://creativecommons.org/licenses/by-nc/4.0/) which permits unrestricted non-commercial use, distribution, and reproduction in any medium, provided the original work is properly cited. 


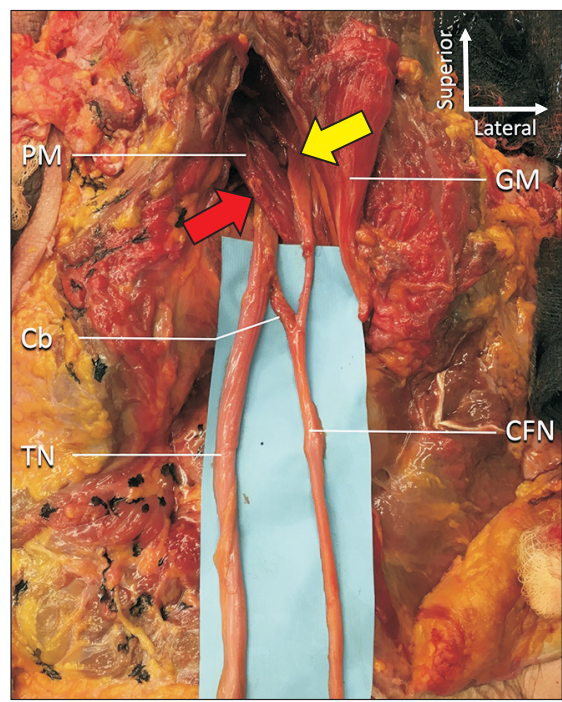

Fig. 1. Gluteal and posterior thigh regions on the right side. The GM is reflected laterally. The superior part (CFN) of the sciatic nerve is seen piercing the PM (yellow arrow) and the inferior root (TN) runs below the inferior border of the PM (red arrow). Note the $\mathrm{Cb}$ between the two. $\mathrm{Cb}$, communicating branch; $\mathrm{CFN}$, common fibular nerve; GM, gluteus maximus; PM, piriformis muscle; TN, tibial nerve.

\section{Discussion}

The manner in which the lumbosacral plexus creates the $\mathrm{SN}$ and how the SN branches into the tibial and CFNs represent variations that can have a significant clinical and therapeutic impact. A study by Antoniadis reported [6] that 25\% of symptomatic SN lesions were iatrogenic, whether through surgical trauma or anesthetic mishaps, highlighting the difficulty in identifying and isolating the $\mathrm{SN}$. Just as Dupont et al. [7] suggest, a thorough survey of a patient's SN course needs to be appreciated prior to treatment in order to minimize the risk of iatrogenic injury, which must be preceeded by an understaning of all the possible $\mathrm{SN}$ variations.

Beyond the Beaton and Anson [2] classification, others have noted different variaitons of the $\mathrm{SN}$ at the piriformis. For example, the passage of the CFN through the infrapiriform foramen, and the $\mathrm{TN}$ under the superior gemellus i.e., a seventh variant [5]. To our knowledge, our cadaver represents a possible eighth variation and the first example where the $\mathrm{SN}$ is split at the PM to then reunite distal to the muscle.

In addition to its relation with the PM, SN variations can also be grouped based on other factors such as its level of bifurcation and its relation with other structures such as blood supply [1]. The SN may even undergo 'trifurcations' where the additional segment becomes a sural nerve or a branch of the perineal nerve [8]. Other times, the ventral rami of L4S3 may not coalesce into one large SN until deep in the lower part of the gluteal region - a variation that can have broad implications in surgery and anesthesiology when conducting nerve blockade [9]. SN divisions proximal to the PM, so called 'high divisions', are not uncommon and can be present on one or both sides [1]. A notable finding is that anatomical variations of the $\mathrm{SN}$ have not been associated with sex [10].

The $\mathrm{SN}$ is involved in conditions such as nondiscogenic sciatica and piriformis syndrome. Symptoms of sciatica include sharp lower back pain that radiates down the posterior leg [11], and piriformis syndrome, characterized by SN entrapment or compression by the PM [12], resulting in pain and tingling from the buttocks to the legs. The different anatomical variations of the $\mathrm{SN}$ can lead to challenges in terms of therapeutic and diagnostic care of these conditions. For example, while there exists numerous locations where the SN can be blocked, some are safer than others. Variations in the anatomy of the $\mathrm{SN}$ can lead to higher rates of incomplete blockade, which can result in continued pain, nerve injury, blood vessel traumatization, and local anesthetic toxicity [13]. For example, Ro and Edmonds presented a case in which a patient with the type B SN arrangement failed to respond to conservative treatment, making surgery the best option [14]. Their case supports the argument that the different variations may require individualized treatment approaches.

In conclusion, an understanding of the different variants of the $\mathrm{SN}$ in regard to the level of division around the PM is of clinical significance. Its functionality and pathology spans several disciplines in medicine such as neurosurgery, neurology, and orthopedics. This case illustration highlights an apparently rare variant of the $\mathrm{SN}$.

\section{ORCID}

Brendan Huang: https://orcid.org/0000-0002-1277-9862

Tess Decater: https://orcid.org/0000-0002-9009-3268

Joe Iwanaga: https://orcid.org/0000-0002-8502-7952

Aaron S. Dumont: https://orcid.org/0000-0002-8077-8992

R. Shane Tubbs: https://orcid.org/0000-0003-1317-1047

\section{Author Contributions}

Conceptualization: JI, RST. Data acquisition: JI, RST. Data analysis or interpretation: BH, TD. Drafting of the manuscript: $\mathrm{BH}$, TD. Critical revision of the manuscript: ASD, 
RST. Approval of the final version of the manuscript: all authors.

\section{Conflicts of Interest}

No potential conflict of interest relevant to this article was reported.

\section{References}

1. Adibatti M, Sangeetha V. Study on variant anatomy of sciatic nerve. J Clin Diagn Res 2014;8:AC07-9.

2. Beaton LE, Anson BJ. The relation of the sciatic nerve and of its subdivisions to the piriformis muscle. Anat Rec 1937;70:1-5.

3. Smoll NR. Variations of the piriformis and sciatic nerve with clinical consequence: a review. Clin Anat 2010;23:8-17.

4. Varenika V, Lutz AM, Beaulieu CF, Bucknor MD. Detection and prevalence of variant sciatic nerve anatomy in relation to the piriformis muscle on MRI. Skeletal Radiol 2017;46:751-7.

5. Güvençer M, Iyem C, Akyer P, Tetik S, Naderi S. Variations in the high division of the sciatic nerve and relationship between the sciatic nerve and the piriformis. Turk Neurosurg 2009;19:139-44.

6. Antoniadis G, Kretschmer T, Pedro MT, König RW, Heinen $\mathrm{CP}$, Richter HP. Iatrogenic nerve injuries: prevalence, diagnosis and treatment. Dtsch Arztebl Int 2014;111:273-9.

7. Dupont G, Unno F, Iwanaga J, Oskouian RJ, Tubbs RS. A variant of the sciatic nerve and its clinical implications. Cureus 2018;10:e2874.

8. Berihu BA, Debeb YG. Anatomical variation in bifurcation and trifurcations of sciatic nerve and its clinical implications: in selected university in Ethiopia. BMC Res Notes 2015;8:633.

9. Gunnal S, Wabale R. An unusual formation of sciatic nerve. Indian J Med Sci 2013;67:193-6.

10. Okraszewska E, Migdalski L, Jedrzejewski KS, Bolanowski W. Sciatic nerve variations in some studies on the Polish population and its statistical significance. Folia Morphol (Warsz) 2002;61:277-82.

11. Khan H, Ling S, Ali S, Jonnalagadda P, Ramsey F, Weiner M, Awan O. Sciatic nerve variants in patients diagnosed with sciatica: is there a correlation? J Comput Assist Tomogr 2019;43:953-7.

12. Robinson DR. Pyriformis syndrome in relation to sciatic pain. Am J Surg 1947;73:355-8.

13. Saleh HA, El-fark MM, Abdel-Hamid GA. Anatomical variation of sciatic nerve division in the popliteal fossa and its implication in popliteal nerve blockade. Folia Morphol (Warsz) 2009;68:256-9.

14. Ro TH, Edmonds L. Diagnosis and management of piriformis syndrome: a rare anatomic variant analyzed by magnetic resonance imaging. J Clin Imaging Sci 2018;8:6. 